\title{
ANALYSIS FOR REFLECTOR ALUMINUM MESH PANELS OF FIVE-HUNDRED METER APERTURE SPHERICAL TELESCOPE *
}

\author{
LI GUOQIANG, SHEN LIYUAN, LUO YONGFENG, DENG CHANGGEN \\ and HE YAMEI \\ College of Civil Engineering, Tongji University, Shanghai, 200092, China
}

\begin{abstract}
In this paper, an analysis of the aluminum reflector mesh panels for the Five-hundred meter Aperture Spherical Telescope (FAST) is described. The study concentrates on one element of the reflector surface with the shape of a triangle. For the analysis, the panel is modelled as a mesh of cables so that it can be treated as continuous. It is demonstrated that the results so obtained are reasonable.
\end{abstract}

Keywords: Aluminum mesh panel, Mesh of cables, Approximate Solution

FAST is a Knowledge Creation Project of the Chinese Academy of Sciences. To understand the behavior of the reflector panels of FAST under environmental loads, a theoretical analysis is carried out in this paper.

A reflector panel of FAST is actually aluminum mesh because of the regular square holes in it. Since the mesh is quite thin $(1 \mathrm{~mm})$, it can only bear tension. There will be great errors in the analysis results if the panel is modelled as a plate without considering membrane stress. In this paper, the panel is treated as a mesh of cables and analyzed by the continuum theory. We demonstrate that our analytical approach provides clearer and more acceptable results.

* This paper is subsidized by the FAST Project of Chinese Academy of Sciences.

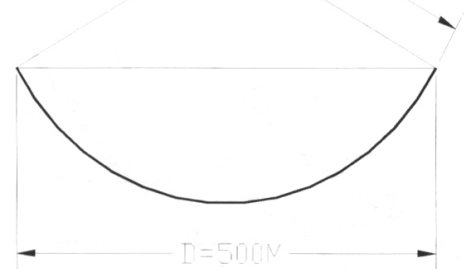

Figure 1. Reflector surface of FAST. 


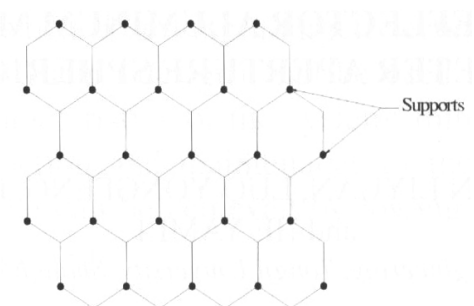

Figure 2. Fundamental elements of the reflector surface

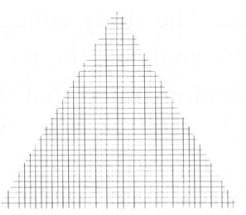

Triangular mesh panel

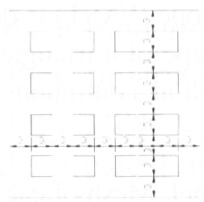

Detail dimensions of mesh

Figure 3. Aluminum triangular mesh panel

\section{Elements of the Reflector Panel}

The reflector surface of FAST is spherical, with a $R=300 \mathrm{~m}$ radius of curvature and $\mathrm{D}=500 \mathrm{~m}$ aperture (Figure 1). The whole surface is divided into many elements which are supported by steel or aluminum grid shells.

The shape of an element is a regular hexagon. Each element is supported by the grid shell at three pointsIJłas shown in Figure 2.

For supporting the reflector aluminum panels, which are only $1 \mathrm{~mm}$ thick, each hexagon is further divided into many triangles of $2.5 \mathrm{~m}$ on a side. Then the fundamental element for analysis of the reflector panel becomes a triangle, as shown in Figure 3 , supported along its edges and with $3 \mathrm{~mm} \times 3 \mathrm{~mm}$ square holes in it.

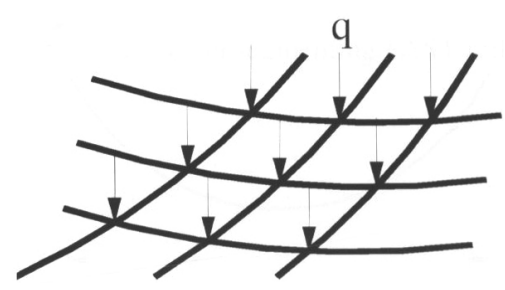

Figure 4. Equivalence of cable mesh. 


\section{Equilibrium Equation}

For analysis, the reflector panel can be modelled as a mesh of cables (Figure 4). There are two methods to analyze this structure: by dispersion relations, and by treating it as a continuum. In this paper, the latter method will be employed.

For utilizing continuum theory, it is supposed that:

1. The cable is ideally flexible;

2. The cable tension conforms to Hooke's law;

3. The cables in the two different directions maintain contact so as to transmit the vertical forces;

4. The deflection of the panel is small and the loads are perpendicular to the panel. By adopting the above assumptions, the following differential increment equilibrium equation of the cable mesh can be established:

$$
\begin{aligned}
& H_{x o} \frac{\partial^{2} \omega}{\partial x^{2}}+H_{y o} \frac{\partial^{2} \omega}{\partial y^{2}}+\Delta H_{x}\left(\frac{\partial^{2} z_{0}}{\partial x^{2}}+\frac{\partial^{2} \omega}{\partial x^{2}}\right) \\
& +\Delta H_{y}\left(\frac{\partial^{2} z_{0}}{\partial y^{2}}+\frac{\partial^{2} \omega}{\partial y^{2}}\right)+\Delta q=0
\end{aligned}
$$

in which

$\omega$ :

the vertical component of displacement;

$Z_{0}=Z_{0}(x, y):$ the initial shape of the cable mesh;

$H_{x 0}, H_{y 0}: \quad$ the conversion of cable pre-tensions in the two directions;

$\Delta H_{x}, \Delta H_{y}: \quad$ the increments of the pre-tensions;

$\Delta q: \quad$ the vertical increment load.

\section{Approximate Solution}

The steps of the approximate solution of equation (1) are as follows:

1. Choose the displacement function $\omega(\mathrm{x}, \mathrm{y})$ according to the boundary requirement:

$$
\omega(x, y)=f_{0}(x, y)+C_{1} f_{1}(x, y)+C_{2} f_{2}(x, y)+\ldots+C_{n} f_{n}(x, y)
$$

in which

$$
\begin{array}{ll}
f_{i}(x, y)(i=0,1,2 \ldots n): & \text { known functions which have been selected; } \\
C_{i}(i=1,2, \ldots n): & \text { coefficients to be obtained; } \\
f_{i}(i=1,2, \ldots n) & \text { equals zero at boundary. }
\end{array}
$$

2. Substituting equation (2) into the left part of equation (1) leads to

$$
\begin{aligned}
& S=H_{x o} \frac{\partial^{2} \omega}{\partial x^{2}}+H_{y o} \frac{\partial^{2} \omega}{\partial y^{2}}+\Delta H_{x}\left(\frac{\partial^{2} z_{0}}{\partial x^{2}}+\frac{\partial^{2} \omega}{\partial x^{2}}\right) \\
& +\Delta H_{y}\left(\frac{\partial^{2} z_{0}}{\partial y^{2}}+\frac{\partial^{2} \omega}{\partial y^{2}}\right)+\Delta q
\end{aligned}
$$




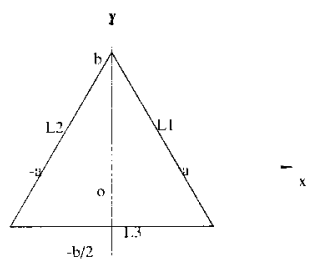

Figure 5. Geometrical parameters of triangular mesh.

The acquired $S$ contains $C_{i}$ and can be expressed as follows:

$$
S=S\left(x, y ; C_{1}, C_{2}, \ldots C_{n}\right)
$$

According to the equilibrium condition, the function $S$ must be zero. Because the assumed function $\omega(x, y)$ is not accurate enough, $S$ will not equal zero in general. Then:

3. Determine the coefficient $C_{i}(i=1,2, \ldots n)$ to make $S$ as small as possible $S$ is required to be orthogonal to each of the selected functions $f_{i}$ :

$$
\iint_{\Omega} S f_{i} d x d y=0(i=1,2, \ldots n)
$$

\section{Analysis of the Reflector Panel}

\subsection{ANALYTICAL PROCEDURE}

When the reflector panel element of FAST is modelled as a cable mesh, two points are noteworthy: (1) the initial curved surface is planar: $z_{0}=z_{0}(x, y)=0$; (2) there is no initial pre-tension in the cables: $H_{x 0}=H_{y 0}=0$. Then the analysis process becomes clear and we can focus upon determining the displacement function.

Set the coordinates system of the cable mesh with respect to the triangular boundary as follows:

The origin of the coordinate system is the centre of the triangle. The sides of the triangular cable mesh are:

$$
L 1: \frac{x}{a}+\frac{y}{b}=1 \quad L 2:-\frac{x}{a}+\frac{y}{b}=1 \quad L 3: y+\frac{b}{2}=0
$$

in which $a=\frac{250}{3}, b=\frac{\sqrt{3}}{3} 2500$

Assume for the vertical displacement function of the triangular cable mesh:

$$
\omega(x, y)=\omega_{0}\left(1-\frac{x}{a}-\frac{y}{b}\right)\left(1+\frac{x}{a}-\frac{y}{b}\right)\left(y+\frac{b}{2}\right)
$$

in which $\omega_{0}$ : coefficient to be confirmed. The vertical displacements at the boundary equal zero.

The following expression can be obtained by the approximate solution $\left(H_{x 0}=\right.$ $H_{y 0}=0$ ) 
TABLE I

The maximum stress of equivalent cables under $Q=0.266 \mathrm{kN} / \mathrm{m}^{2}$

\begin{tabular}{lllc}
\hline $\mathrm{q}=0.266 \mathrm{kN} / \mathrm{m}^{2}$ & \multirow{2}{*}{$\omega_{0}(\mathrm{~mm})$} & \multicolumn{2}{c}{ maximum stress $\left(\mathrm{N} / \mathrm{mm}^{2}\right)$} \\
\cline { 3 - 4 } & & $X$ direction & $Y$ direction \\
\hline Neglecting the thermal effect & 0.0180 & 11.83 & 7.98 \\
Including thermal effect $\left(\Delta T=30^{\circ} \mathrm{C}\right)$ & 0.0499 & 39.11 & 9.58 \\
Including thermal effect $\left(\Delta T=-30^{\circ} \mathrm{C}\right)$ & 0.0025 & 52.00 & 51.93 \\
\hline
\end{tabular}

TABLE II

The maximum deflection of equivalent cables under $Q=0.014 \mathrm{kN} / \mathrm{m}^{2}$

\begin{tabular}{lll}
\hline $\mathrm{q}=0.014 \mathrm{kN} / \mathrm{m}^{2}$ & $\omega_{0}(\mathrm{~mm})$ & maximum deflection $(\mathrm{mm})$ \\
\hline Neglecting the thermal effect & 0.0068 & 4.91 \\
Including thermal effect $\left(\Delta T=30^{\circ} \mathrm{C}\right)$ & 0.0488 & 35.22 \\
\hline
\end{tabular}

$$
s=s\left(x, y ; \omega_{0}\right)
$$

The equation of variation is:

$$
\iint_{\Omega} S\left(x, y ; \omega_{0}\right)\left(1-\frac{x}{a}-\frac{y}{b}\right)\left(1+\frac{x}{a}-\frac{y}{b}\right)\left(y+\frac{b}{2}\right) d x d y=0
$$

The equation for $\omega_{0}$ can be obtained from the integral of equation (7), and the cable tension can be determined. The maximum deflection of the cable mesh at its centre, $b \omega_{0} / 2$, and the forces in the cables can be obtained from the value of $\omega_{0}$.

\subsection{RESULTS OF ANALYSIS}

Two groups of loads including thermal effects are considered for studying the behavior of a FAST reflector panel. The results are listed in Table I and Table II.

\section{Conclusion}

In this paper, the reflector surface of FAST is modelled as a cable mesh, using a continuum approach. The numerical results show that the vertical loads are resisted mainly by tension in the assumed cables. It is demonstrated that modelling the panel as a cable mesh is more practical and accurate than taking it to be a plate. 
The membrane elements will be used in future research and the results obtained here will be extended.

\section{References}

Guo, Lin: 1987, Mathematical Method of Engineering, Published by the Press of Mechanical Industry, $1^{\text {st }}$ edition in February, 1987.

Guoqiang, Li and Yongfeng, Luo: The $\Phi 500 \mathrm{M}$ Giant Spherical Telescope and its Key points of Structure, Communication Material of FAST.

Shizhao, Shen, et al.: 1997, Design of Cable Structure, Published by the Press of Chinese Architectural Industry, $1^{\text {st }}$ edition in August, 1997.

Yuhai, Qiu: 1998, The Giant Spherical Radio Telescope with The Main Active Reflecting Surface. Study of Astrophysics 18(2), 222-227. 